\title{
BMJ Open Mediators and theories of change in psychotherapy with adolescents: a systematic review protocol
}

\author{
Svenja Taubner (D) , ${ }^{1}$ Andrea Saliba, ${ }^{2}$ Erkki Heinonen (D) , ${ }^{3,4}$ Sonja Protić, ${ }^{5}$ \\ Jana Volkert (i) , ${ }^{1}$ Asta Adler, ${ }^{6}$ Rasa Barkauskiene (1) , ${ }^{6}$ Sonia Conejo Cerón (1) ,7,8 \\ Dina Di Giacomo (D) , 9 Yianna loannou (D) ,10 Jose M Mestre (D) , 11 \\ Patricia Moreno-Peral (1) , 8,12 Filipa Mucha Vieira, ${ }^{13}$ Catarina Pinheiro Mota, ${ }^{13,14}$ \\ Marija Raleva, ${ }^{15}$ Margarida Isabel Rangel Santos Henriques, ${ }^{16}$ Jan Ivar Røssberg, ${ }^{17}$ \\ Stefanie J. Schmidt, ${ }^{18}$ Tjaša Stepišnik Perdih, ${ }^{19}$ Randi Ulberg, ${ }^{17,20}$ \\ Célia M D Sales (i) ${ }^{13}$
}

To cite: Taubner S, Saliba A, Heinonen $\mathrm{E}$, et al. Mediators and theories of change in psychotherapy with adolescents: a systematic review protocol. BMJ Open 2021;11:e042411. doi:10.1136/ bmjopen-2020-042411

- Prepublication history and additional materials for this paper is available online. To view these files, please visit the journal online (http://dx.doi. org/10.1136/bmjopen-2020042411).

Received 06 July 2020 Revised 17 November 2020 Accepted 24 February 2021

Check for updates

(C) Author(s) (or their employer(s)) 2021. Re-use permitted under CC BY-NC. No commercial re-use. See rights and permissions. Published by BMJ.

For numbered affiliations see end of article.

Correspondence to Dr Svenja Taubner, Institute for Psychosocial Prevention, University Heidelberg, Heidelberg, Germany; svenja.taubner@med.uniheidelberg.de

\section{ABSTRACT}

Introduction Approximately $75 \%$ of mental disorders emerge before the age of 25 years but less than half receive appropriate treatment. Little is known about the mechanisms underlying the therapeutic change of adolescents in psychotherapy. The 'European Network of Individualised Psychotherapy Treatment of Young People with Mental Disorders', funded by the European Cooperation in Science and Technology, will conduct the first systematic review to summarise the existing knowledge on mediators and theories of change in psychotherapy for adolescents.

Method A systematic review will be conducted, conforming to the reporting guidelines of the Preferred Reporting Items for Systematic Reviews and MetaAnalyses statement recommendations. Electronic databases (PubMed and PsycINF0) have been systematically searched on 23 February 2020 , for prospective, longitudinal and case-control designs which examine mediators of change. Participants will be adolescents between 10 and 19 years of age who suffer from a mental disorder or psychological difficulties and receive an intervention that aims at preventing, ameliorating and/or treating psychological problems. Ethics and dissemination Ethical approval is not required for this systematic review as no primary data will be collected. The results will be published in a peerreviewed journals and at conference presentations and will be shared with stakeholder groups. The whole data set will be offered to other research groups following recommendations of the open science initiative. Databases with the systematic search will be made openly available following open science initiatives.

PROSPERO registration number CRD42020177535.

\section{INTRODUCTION}

Adolescents and young adults are a particularly important and vulnerable group with distinct mental health needs. According to Kessler et al, $50 \%$ of lifetime diagnosable mental health disorders start by the age of

\section{Strengths and limitations of this study}

- Review aims at identifying underlying mechanisms of change in the process of psychotherapy for adolescents.

- Reviewers are from a European multidisciplinary researcher network with researchers and clinicians from 30 countries.

- First comprehensive overview of mediators in psychotherapy carried out with adolescents.

- Broad inclusion criteria increase external validity but limits the possibility of causal conclusions including non-randomised controlled trials studies, it is likely not feasible to estimate aggregated effect sizes for the identified mediators.

14 , and this number increases to $75 \%$ by the age of $25 .{ }^{12}$ Ignoring young people's mental health needs can result in long-lasting adverse developmental outcomes for the individual and society. These include: psychiatric disorders in adulthood, impaired social functioning, difficulties with employment as well as poorer general health outcomes. ${ }^{3}$

Psychotherapy is effective, efficient and costeffective, providing long-term impacts which are not possible with psychopharmacological treatment alone. ${ }^{4-6}$ There is strong evidence on the general effectiveness of psychotherapy for treating mental disorders in adolescents, ${ }^{78}$ and a wealth of psychotherapy treatment modalities are currently being used and researched, with $>550$ different psychotherapy models that can be applied to young people. ${ }^{9}$ Treatment response is explained by different mechanisms of change and accounted for by a variety of mediators (eg, self-reflection, learning compensatory skills, changing automatic thoughts and so on) 
associated with each treatment modality. While various psychotherapy modalities (eg, psychodynamic psychotherapy; cognitive behavioural therapy) have shown equally good treatment outcomes overall, adherence to a specific treatment modality has not shown to significantly impact the outcome in child and adolescent psychotherapy. ${ }^{10}$ However, to understand therapeutic change and enhance outcomes, it is necessary to identify treatment processes or characteristics within the therapist, the adolescent, parent or family that facilitate successful therapeutic change and isolate those that are redundant and can be dismissed. ${ }^{9}$ Thus, research on underlying specific mechanisms of change across treatment modalities and settings is essential to optimising treatment.

An important first step towards examination of mechanisms of change in psychotherapy is the identification of mediators. While moderators (eg, gender, age) serve to clarify what kind of treatment is adequate for a specific person under certain circumstances, mechanisms of change define causal relationships between therapeutic change and psychological interventions. A mechanism of change explains how an intervention translates into a process that leads to an outcome, for example, change in symptoms. ${ }^{11}$ Thus, a mechanism is an explanatory concept that can be investigated by researching mediators, that is, variables that explain changes statistically. Kazdin has formulated clear criteria on how to assess mediators of psychological treatments ${ }^{12}$ :

- Conduct sufficiently powered randomised clinical trials.

- Use valid and reliable measures for mediators that are sensitive to change.

- Apply a process design in which changes of the mediator temporally precede changes in therapeutic outcome and the mediator variable is measured repeatedly.

- Compare mediators that are theory-driven with nonspecific mediators.

- Apply different dosages to prove that a stronger mediator-change leads to more therapeutic changes.

However, even after 13 years of Kazdin's suggestions on how to assess mechanisms of change in psychotherapy research, ${ }^{12}$ there are still an alarming lack of studies, inconclusive results (eg, the treatment of depression in adults) ${ }^{13}$ and very little research on change mechanisms for the treatment of adolescents. ${ }^{14} 15$ Cuijpers $e t$ $a l$ conclude that despite $>70$ years of systematic psychotherapy research, we have no empirically validated mechanisms of change in adult psychotherapy. ${ }^{16}$ The current systematic review will be the first to summarise the existing knowledge on mediators and theories of change in psychotherapy for adolescents.

To address these challenges related to mediators and measures of the mechanisms of change and therapies' efficacy in children and adolescents, besides other activities, the European Cooperation in Science and Technology (COST) funded a 4-year programme named 'European Network of Individualised Psychotherapy Treatment of
Young People with Mental Disorders' (TREATme) (www. treat-me.eu) that serves as a European multidisciplinary researcher network with researchers and clinicians from 30 countries. TREATme will review the academic research relating to mechanisms of change in patients aged between 10 and 30 years receiving psychological treatments. For the current review, the part of the collected data set concerning adolescents only will be used. The aim is to provide an overview of existing research on psychological factors that mediate psychotherapeutic change in adolescents. We will conduct a narrative synthesis of all studies available up to 2020. The objectives of this review are the following:

1. To identify which mediators and theories of change have been studied in psychotherapy with adolescents.

2. To identify if there are adolescence-specific, disorderspecific or treatment-specific mediators.

3. To critically evaluate the methodological approach of the current research data available on mediators in psychotherapy for adolescents.

In order to obtain a comprehensive overview of the field of psychotherapy for adolescents, we will include various forms of psychotherapy and quantitative research designs. We will select all studies including a statistical test of mediation (eg, Baron and Kenny or more advanced methods ${ }^{17}$ and will summarise study characteristics and results. We will discuss the extent to which these studies meet the most important requirements for mediator research that were mentioned earlier. Furthermore, we will relate mediators to the respective theories of change and describe which mediators successfully explain therapeutic change. Ultimately, this review can contribute to the challenging process of identifying underlying mechanisms of change in the process of psychotherapy for adolescents.

\section{METHOD}

The review follows the Preferred Reporting Items for Systematic Review and Meta-Analysis Protocols. ${ }^{18}$ This article is based on work from the COST Action TREATme aiming to improve knowledge and understanding of psychotherapeutic interventions in young people. The patient/population, intervention, comparison and outcomes (PICO) model was used to define the research question.

\section{Eligibility criteria}

Studies from any geographical location, written in English, available as full-text and published from inception onwards until 23 February 2020 that meet criteria, will be included in the review. Grey literature such as theses, dissertations or conference proceedings will also be included.

\section{Types of studies}

Due to a lack of studies fulfilling all criteria for mediator research, we decided to include a broad range of study 
types. Studies will be selected if they include statistical analysis of mediators in psychotherapy. This comprises (a) empirical quantitative studies following prospective, longitudinal and case-control designs, (b) terms related to or describing mediators and (c) a psychosocial intervention and/or psychotherapeutic intervention or treatment for primary/secondary prevention.

\section{Types of participants}

WHO defines adolescents as individuals between 10 and 19 years of age, ${ }^{19}$ which was the age group selected for our inclusion criteria. These participants would have a mental disorder (eg, depression, eating disorders) or psychological difficulties (eg, binge drinking) and receive a psychotherapeutic intervention, including primary and secondary prevention programmes. All comparators will be included as we will be investigating mediators in all treatments and not the efficacy of one treatment over another.

\section{Types of interventions}

Studies will be included if they report an intervention aimed at preventing, ameliorating and/or treating psychological problems of adolescents by using psychosocial mechanisms and strategies in any setting (ie, individual, family, group, inpatients, eMental health and so on). These interventions should not be primarily biological or physiological. Examples of interventions include all branches or types of psychotherapy: psychodynamic, integrative, systemic, cognitive-based or cognitive-behavioural, interpersonal, humanistic (such as emotion-focused, supportive, motivational interviewing), psychoeducation and third-wave approaches (such as mindfulness-based therapies).

\section{Search strategy}

The search strategy includes terms relating to or describing the intervention. These terms have been combined with the Cochrane MEDLINE filter for controlled trials of interventions and were adapted for PsycINFO. ${ }^{20}$ Studies published from inception to 23 February 2020 have been sought. The search string can be found in the online supplemental file 1 . The search was performed on 23 February 2020.

\section{Data extraction}

Study selection will be carried out by a group of 20 experienced researchers divided into 10 pairs who will independently assess the eligibility of studies retrieved using the search strategy in two phases. The first phase comprises the screening of the titles and/or abstracts of studies that potentially meet the inclusion criteria outlined above. In the second phase, each pair of reviewers will evaluate the full text of these potentially eligible studies to see if they meet the inclusion criteria. Disagreements will be discussed by the pair, and a third reviewer will be involved if consensus cannot be reached. Finally, a fourth independent reviewer will perform an additional quality control check by assessing the eligibility of every fifth excluded study. Disagreements at this stage will be solved through discussion with the original review pair.

Data records will be managed with the support of Microsoft Excel. ${ }^{21} \mathrm{~A}$ standardised form will be used to extract the information for the review. Extracted information will include: study setting; study population, participant demographics and baseline characteristics; details of the intervention and control conditions; study methodology; outcomes and times of measurement; assessed mediators; type of mediation analysis and information for assessment of the risk of bias. Two review authors will extract information independently; discrepancies will be identified and resolved through discussion or with a third author when necessary.

\section{Critical appraisal}

Although no standard form for evaluating mediation studies has been established, studies will be checked against general criteria for identifying mediators of psychosocial interventions in research, such as summarised by $\operatorname{Kazdin}^{12}$ and Lemmens et al. ${ }^{13}$

\section{Data synthesis}

We will provide a narrative synthesis of the findings from the included studies, with a focus on the categories of mediators that have been tested, types of psychosocial interventions that have been investigated and mental disorders or psychological difficulties of adolescents that have been treated. It will be discussed if age-specific, disorder-specific or treatment-specific mediators can be identified. The age-specific mediators will be identified by comparing results with mediators identified by reviews from adult psychotherapy.

\section{Outcomes and prioritisation}

The primary outcome will be mediators related to change in mental health problems or disorders in adolescents measured by a validated instrument or tool made for that purpose. Data on the disorder will be categorised into depression, anxiety, substance misuse, externalising problems, severe mental illness (eg, psychosis, bipolar disorder) and others (eg, eating disorders). The therapies will be categorised into: psychodynamic, integrative, systematic, cognitive-based or cognitive-behavioural, interpersonal, humanistic, psychoeducation and thirdwave. Mediators and instruments for assessing them will be identified. The statistical analyses for evaluating the effect of mediators will be recorded. Pretreatment and post-treatment scores will be noted, and the differences compared.

\section{Risk of bias in individual studies}

The Mixed Methods Appraisal Tool will be used to evaluate the overall study quality using a formal risk of bias assessment. ${ }^{22}$ This tool permits the appraisal of the methodological quality of five categories of studies: qualitative research, randomised controlled trials, non-randomised studies, quantitative descriptive studies and mixed methods studies. 


\section{Patient and public involvement}

No patient was involved in planning and conducting the study.

\section{Ethics and dissemination}

No ethical approval will be necessary to conduct the review as no additional empirical data will be assessed. Results from the review will be disseminated through a publication in a peer-reviewed open access journal and through scientific communication using the COST Action homepage. The data set that will be established during the search and extractions will be published in an open data repository after the acceptance of the publication of the review order to facilitate access to students, academia and professionals. Additionally, results will be disseminated in conferences, seminars, congresses or symposia.

\section{DISCUSSION}

As far as the authors are aware, this is the first systematic review of its kind, assessing mediators of psychotherapeutic changes in adolescents. The findings will inform how much is currently known about such mediators and the practical implications of this knowledge for treatment planning and outcomes. The results will also shed light on how these empirically studied mediators correspond with the theoretically putative mechanisms of change in particular models of intervention. Using qualitative synthesis, we will evaluate and comment on the conclusiveness of age-specific, disorder-specific or treatmentspecific mediators and map out the most pressing needs for future research on mediators and mechanisms of change in adolescent psychotherapy. The review will provide a basis for comparing our results with those of systematic reviews in adults, to assess whether there are similar or different mediators in adolescents that explain therapeutic change.

The strengths of this protocol include the gathering of a large multidisciplinary group of international researchers with long-standing accumulated experience in the area that have worked on this topic for 3 years through regular online and face-to-face meetings. Furthermore, the group has consulted international experts in the field to develop the protocol. A structured quality assurance will be carried out, as well as several search updates to ensure the completeness of the data set. The whole data set will be offered to other research groups following the recommendations of the open science initiative.

Limitations of this protocol include the use of broad inclusion criteria to describe the existing knowledge comprehensively and increase external validity, which likely limits the possibility of causal conclusions by also including non-randomised controlled trials. For the same reason, it is likely not feasible to estimate aggregated effect sizes for the identified mediators. Our conclusions on mechanisms of change will only be related to empirical quantitative studies as we have excluded qualitative and theoretical studies. As there is no generally accepted gold standard for mediation analysis, we expect much variance in the studies, which could lead to our results being inconclusive or inconsistent. Also, we are analysing both subclinical conditions and diagnosable disorders, as well as intervention and prevention studies, which may lead to less consistent or coherent results. We have chosen to do this in order to address the adolescent age group holistically and aim to report the results separately in order to establish independent conclusions. As the rater team consists of a fairly large group, inter-rater bias has to be strictly monitored, and so a fourth independent reviewer will be introduced to perform additional quality control checks.

\section{Author affiliations}

${ }^{1}$ Institute for Psychosocial Prevention, University Heidelberg, Heidelberg, Germany ${ }^{2}$ Mental Health Services Malta, University of Malta, Msida, Malta

${ }^{3}$ Department of Psychology, University of Oslo, Oslo, Norway

${ }^{4}$ Finnish Institute for Health and Welfare, Helsinki, Finland

${ }^{5}$ Institute of Criminological and Sociological Research, Belgrade, Serbia

${ }^{6}$ Institute of Psychology, Vilnius University, Vilnius, Lithuania

${ }^{7}$ Prevention and Health Promotion Research Network (redIAPP), ISCIII, Málaga, Spain

${ }^{8}$ Instituto de Investigación Biomédica de Málaga, IBIMA, Málaga, Spain

${ }^{9}$ Department of Life, Health and Enviromental Sciences, University of L'Aquila, L'Aquila, Italy

${ }^{10}$ Department of Social Sciences, University of Nicosia, Nicosia, Cyprus

${ }^{11}$ Department of Psychology, University of Cadiz, Cadiz, Spain

${ }^{12}$ Primary Care Prevention and Health Promotion Network (redIAPP), Malaga, Spain

${ }^{13}$ Faculty of Psychology and Education Sciences at University of Porto (FPCEUP), Center for Psychology at University of Porto (CPUP), University of Porto, Porto,

Portugal

${ }^{14}$ Department of Psychology, University of Trás-os-Montes and Alto Douro, Vila Real, Portugal

${ }^{15}$ Institute for Marriage, Family and Systemic Practice—ALTERNATIVA, Skopje, The Former Yugoslav Republic of Macedonia

${ }^{16}$ Department of Psychology, University of Porto, Porto, Portugal

${ }^{17}$ Institute of Clinical Medicine, University of Oslo, Oslo, Norway

${ }^{18}$ Department of Clinical Psychology and Psychotherapy, University of Bern, Bern, Switzerland

${ }^{19}$ School of Advanced Social Studies, Nova Gorica, Slovenia

${ }^{20}$ Department of Psychiatry, Diakonhjemmet Hospital, Oslo, Norway

\section{Twitter Stefanie J. Schmidt @child}

Contributors All coauthors provided a substantial contribution to the conception and design of the work by developing the research question, the search string and carrying out the stage 1 screening (ST, AS, EH, SP, JV, AA, RB, SCC, DDG, YI, JMM, PM-P, FMV, CPM, MR, MIRSH, JIR, SS, TSP, RU, CMDS). The current manuscript was drafted by the first and second as well as the last author (ST, AS, CMDS) and was corrected and finally approved by the other authors (EH, SP, JV, AA, RB, SCC, DG, YI, JM, PM-P, FMV, CPM, MR, MIRSH, JIR, SJS, TSP, RU). RU coordinates the overall COST initiative. All authors agree to be accountable for all aspects of the work in ensuring that questions related to the accuracy or integrity of any part of the work are appropriately investigated and resolved.

Funding This article is based on work from COST Action TREATme (CA 16102), supported by COST (European Cooperation in Science and Technology) (www.cost. eu).

Competing interests None declared.

Patient consent for publication Not required.

Provenance and peer review Not commissioned; externally peer reviewed.

Supplemental material This content has been supplied by the author(s). It has not been vetted by BMJ Publishing Group Limited (BMJ) and may not have been peer-reviewed. Any opinions or recommendations discussed are solely those of the author(s) and are not endorsed by BMJ. BMJ disclaims all liability and responsibility arising from any reliance placed on the content. Where the content includes any translated material, BMJ does not warrant the accuracy and reliability 
of the translations (including but not limited to local regulations, clinical guidelines, terminology, drug names and drug dosages), and is not responsible for any error and/or omissions arising from translation and adaptation or otherwise.

Open access This is an open access article distributed in accordance with the Creative Commons Attribution Non Commercial (CC BY-NC 4.0) license, which permits others to distribute, remix, adapt, build upon this work non-commercially, and license their derivative works on different terms, provided the original work is properly cited, appropriate credit is given, any changes made indicated, and the use is non-commercial. See: http://creativecommons.org/licenses/by-nc/4.0/.

\section{ORCID iDs}

Svenja Taubner http://orcid.org/0000-0001-8058-762X

Erkki Heinonen http://orcid.org/0000-0001-8846-8983

Jana Volkert http://orcid.org/0000-0003-1555-2599

Rasa Barkauskiene http://orcid.org/0000-0002-4464-2481

Sonia Conejo Cerón http://orcid.org/0000-0002-1357-2281

Dina Di Giacomo http://orcid.org/0000-0001-8189-2052

Yianna loannou http://orcid.org/0000-0002-1241-904X

Jose M Mestre http://orcid.org/0000-0002-6822-4970

Patricia Moreno-Peral http://orcid.org/0000-0003-4130-9090

Célia M D Sales http://orcid.org/0000-0002-2586-8469

\section{REFERENCES}

1 Kessler RC, Avenevoli S, Costello EJ, et al. Prevalence, persistence, and sociodemographic correlates of DSM-IV disorders in the National comorbidity survey replication adolescent supplement. Arch Gen Psychiatry 2012;69:372-80.

2 Kessler RC, Berglund P, Demler O, et al. Lifetime prevalence and age-of-onset distributions of DSM-IV disorders in the National comorbidity survey replication. Arch Gen Psychiatry 2005;62:593-602.

3 Patton GC, Sawyer SM, Santelli JS, et al. Our future: a lancet commission on adolescent health and wellbeing. Lancet 2016;387:2423-78

4 Cuijpers P, Sijbrandij M, Koole SL, et al. The efficacy of psychotherapy and pharmacotherapy in treating depressive and anxiety disorders: a meta-analysis of direct comparisons. World Psychiatry 2013;12:137-48.

5 Cuijpers P, Karyotaki E, de Wit L, et al. The effects of fifteen evidence-supported therapies for adult depression: a meta-analytic review. Psychother Res 2020;30:279-93.

6 Woll CFJ, Schönbrodt FD. A series of meta-analytic tests of the efficacy of long-term Psychoanalytic psychotherapy. Eur Psychol 2020;25:51-72.
7 Zhou X, Hetrick SE, Cuijpers P, et al. Comparative efficacy and acceptability of psychotherapies for depression in children and adolescents: a systematic review and network meta-analysis. World Psychiatry 2015;14:207-22.

8 Zhou X, Zhang Y, Furukawa TA, et al. Different types and acceptability of psychotherapies for acute anxiety disorders in children and adolescents: a network meta-analysis. JAMA Psychiatry 2019;76:41.

9 Kazdin AE. Psychotherapy for children and adolescents. Annu Rev Psychol 2003;54:253-76.

10 Collyer H, Eisler I, Woolgar M. Systematic literature review and metaanalysis of the relationship between adherence, competence and outcome in psychotherapy for children and adolescents. Eur Child Adolesc Psychiatry 2020;29:417-31.

11 Elliott R. Psychotherapy change process research: realizing the promise. Psychother Res 2010;20:123-35.

12 Kazdin AE. Mediators and mechanisms of change in psychotherapy research. Annu Rev Clin Psychol 2007;3:1-27.

13 Lemmens LHJM, Müller VNLS, Arntz A, et al. Mechanisms of change in psychotherapy for depression: an empirical update and evaluation of research aimed at identifying psychological mediators. Clin Psychol Rev 2016;50:95-107.

14 Kazdin AE, Nock MK. Delineating mechanisms of change in child and adolescent therapy: methodological issues and research recommendations. J Child Psychol Psychiatry 2003;44:1116-29.

15 Schmidt SJ, Schimmelmann BG. Fortschritte, Herausforderungen und neue Perspektiven Der Psychotherapieforschung bei Kindern und Jugendlichen. Z Kinder Jugendpsychiatr Psychother 2015;43:85-90

16 Cuijpers P, Reijnders M, Huibers MJH. The role of common factors in psychotherapy outcomes. Annu Rev Clin Psychol 2019;15:207-31.

17 Baron RM, Kenny DA. The moderator-mediator variable distinction in social psychological research: conceptual, strategic, and statistical considerations. J Pers Soc Psychol 1986;51:1173-82.

18 Moher D, Shamseer L, Clarke M, et al. Preferred reporting items for systematic review and meta-analysis protocols (PRISMA-P) 2015 statement. Syst Rev 2015;4:1-9.

19 World Health Organisation. Adolescent health, 2020. Available: https://www.who.int/health-topics/adolescent-health/

20 Higgins JPT, Thomas J, Chandler J, eds. Cochrane handbook for systematic reviews of interventions version 6.0, 2019. www.training. cochrane.org/handbook

21 Microsoft Corporation. Microsoft Excel, 2020. Available: https:// office.microsoft.com/excel

22 Hong QN, Fàbregues S, Bartlett G, et al. The mixed methods appraisal tool (MMAT) version 2018 for information professionals and researchers. EFI 2018;34:285-91. 